This item was submitted to Loughborough's Research Repository by the author.

Items in Figshare are protected by copyright, with all rights reserved, unless otherwise indicated.

\title{
Optimisation-based verification process of obstacle avoidance systems for unicycle-like mobile robots
}

\section{PLEASE CITE THE PUBLISHED VERSION}

http://link.springer.com/article/10.1007/s11633-011-0590-4

\section{PUBLISHER}

(c) Springer Verlag and Institute of Automation, Chinese Academy of Sciences

\section{VERSION}

AM (Accepted Manuscript)

\section{LICENCE}

CC BY-NC-ND 4.0

\section{REPOSITORY RECORD}

Srikanthakumar, Sivaranjini, and Wen-Hua Chen. 2012. "Optimisation-based Verification Process of Obstacle Avoidance Systems for Unicycle-like Mobile Robots”. figshare. https://hdl.handle.net/2134/11133. 
This item was submitted to Loughborough's Institutional Repository (https://dspace.lboro.ac.uk/) by the author and is made available under the following Creative Commons Licence conditions.

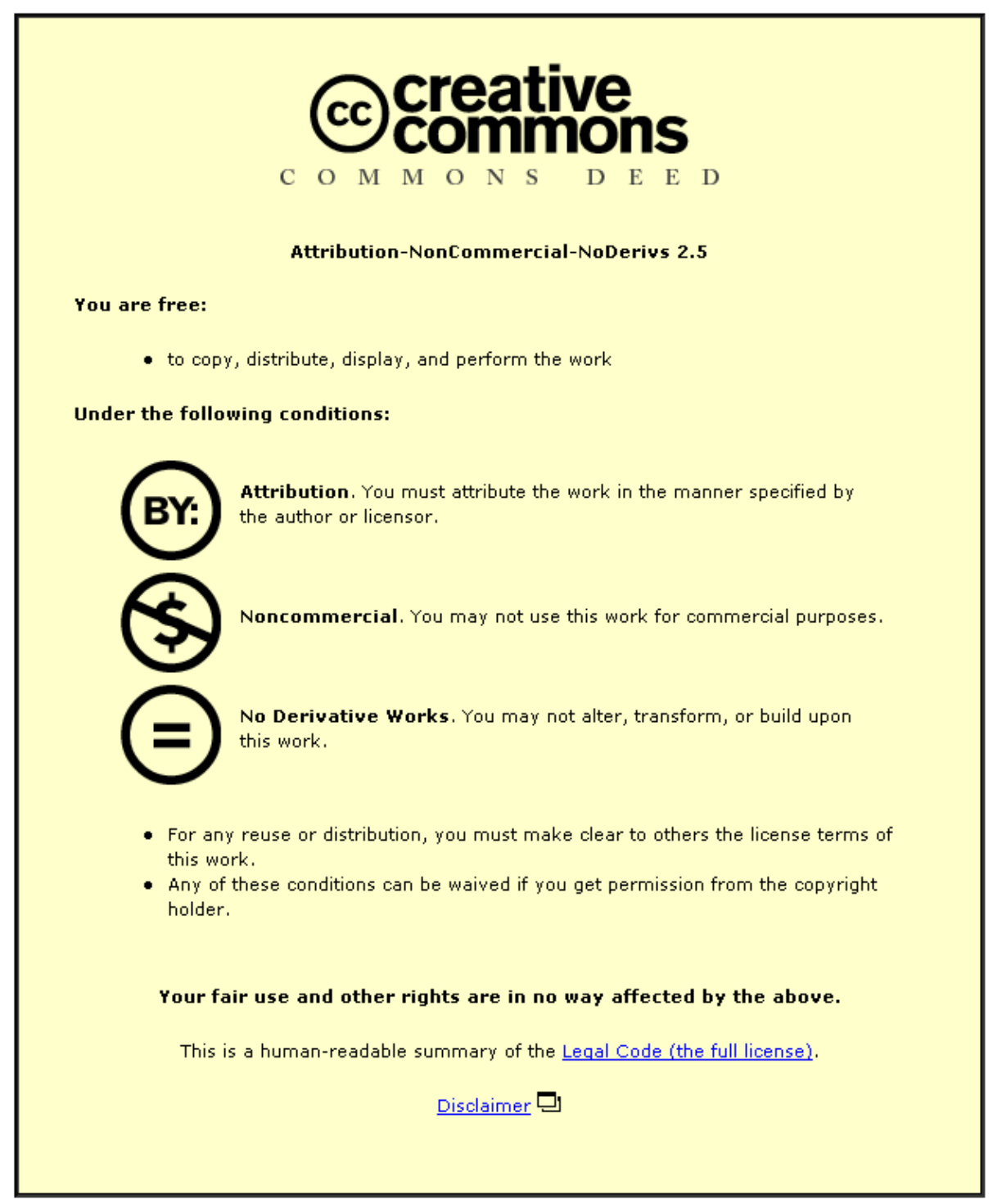

For the full text of this licence, please go to: http://creativecommons.org/licenses/by-nc-nd/2.5/ 


\title{
Optimisation-based Verification Process of Obstacle Avoidance
}

\author{
Systems for Unicycle-like Mobile Robots
}

\author{
Sivaranjini Srikanthakumar, Wen-Hua Chen \\ Department of Aeronautical and Automotive Engineering, Loughborough University, LE11 3TU, UK
}

\begin{abstract}
This paper presents an optimisation-based verification process for obstacle avoidance systems of unicycle-like mobile robot. It is a novel approach for collision avoidance verification process. Local and global optimisation based verification processes are developed to find the worst-case parameters and the worst-case distance between the robot and an obstacle. The kinematic and dynamic model of the unicycle-like mobile robots is firstly introduced with force and torque as the inputs. The design of the control system is split in two parts. One is velocity and rotation using the robot dynamics and the other is the incremental motion planning for robot kinematics. The artificial potential field method is chosen as a path planning and obstacle avoidance candidate technique for verification study as it is simple and widely used. Different optimisation algorithms are applied and compared for the purpose of verification. It is shown that even for a simple case study where only mass and inertia variations are considered, a local optimization based verification method may fail to identify the worst case. Two global optimization methods have been investigated: Genetic Algorithms (GA's) and GLOBAL algorithms. Both these two methods successfully find the worst case. The verification process confirms that the obstacle avoidance algorithm functions correctly in the presence of all the possible parameter variations.
\end{abstract}

Keywords: Verification Process, Obstacle Avoidance, Unicycle Mobile Robot, Potential Field Method, Optimisation.

\section{Introduction}

Autonomous vehicle technology is being rapidly applied to many applications for daily civilian and military life. Motion planning, including collision avoidance, is a very important subject in the development of autonomous vehicles. Indeed, a significant amount of research has been devoted to this subject in recent years. The main goal of this study is to develop advanced algorithms to support safety-critical obstacle avoidance systems in mobile robotics. Without a driver, computer algorithms must be developed to generate a feasible path in real time. Depending on the operation scenarios, there are different kinds of path planning methods. The robot has to find a collision-free path between the start and the goal configurations in a static and a dynamic environment containing various obstacles. A static circular obstacle in a clearly known environment is considered for this study. Several algorithms have been developed for robot path planning in the presence of known obstacles. These algorithms include graph search methods, probabilistic road map planners, cell-decomposition, and potential field methods ${ }^{[1]}$. In this paper, potential filed method is chosen for the local holonomic planner.

The artificial potential field method is a very well known approach for the control and path planning and was originally developed by Khatib, 1986 for manipulators and mobile robots as a local method ${ }^{[2]}$.

Manuscript received date ; revised date
With artificial potential filed methods, the robot moves under the local effects of repulsive fields associated to obstacles and an attractive field pulling toward the goal. In local path planning methods a particular attention is paid to local minima problem. This problem occurs when a robot navigates towards a desired target with no priori knowledge of the environment and gets trapped in a loop ${ }^{[3]}$. Several modifications have been introduced to over-come this problem ${ }^{[4,5]}$.

Three major concerns in regard to autonomous vehicle operation are efficiency, safety and accuracy. As the safety of the mobile robot is dependent on the controller and the obstacle avoidance algorithm, it must be proven that the controller and obstacle avoidance algorithm function correctly in the presence of all possible vehicle and environmental variations. Two particular difficulties faced by designers are nonlinearity and uncertainty in the autonomous vehicle dynamics ${ }^{[6]}$. The verification process is to prove that the vehicle is safe under all conditions and variations. This means that the work must be performed not only for the nominal model, but also for all possible vehicle and environmental variations. Therefore, the model and algorithm has to be extended to analysis of such uncertainties. All possible combinations of robot parameters must be investigated so that guarantees about the worst-case performance can be made. This verification and validation task is a very time consuming and expensive process.

In this study, the minimum distance to the obstacle is considered as the objective function in the time domain with subject to the uncertain parameter bounds. Therefore, the minimum distance to the obstacle $\left(d_{\text {min }}\right)$ must be greater than the radius of obstacle $(r)$ including safe margin. For an anti-collision system, $d_{\min }$ must be greater than $r$ $\left(d_{\min }>r\right)$ in the presence of all the uncertain parameter 


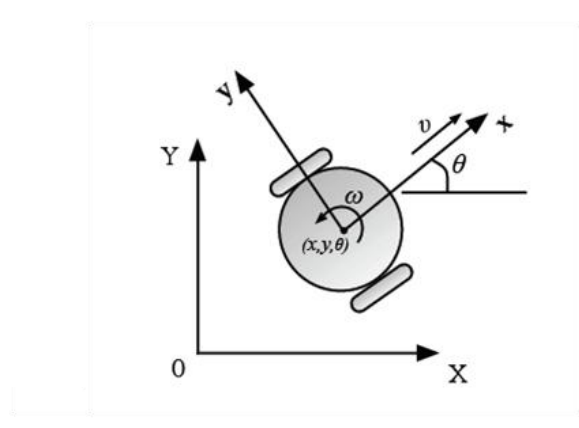

Figure 1. A Unicycle Mobile Robot

variations. Otherwise, the obstacle avoidance algorithm and controller have to be refined to satisfy the anti-collision specification. In [7], fault tree analysis method was applied to the TCAS (Traffic Alert and Collision Avoidance Systems) for the safety analysis.

This paper advocates an optimization based verification approach for mobile robots. To the best of our knowledge, it is the first time that optimisation based verification process has been studied for the collision avoidance systems. Local and global optimisation methods are used to search simultaneously for the worst case condition (minimum distance to the obstacle) and the worst case uncertain parameter combination for the two wheeled mobile robot.

The rest of the paper is organized as follows: the kinematic and dynamic model of the unicycle robot is introduced in Section 2. Motion control and collision avoidance algorithm designed using artificial potential filed methods is presented in Section 3. In Section 4, initial robustness analysis of the collision avoidance algorithm is studied. Optimisation based verification process is proposed and local optimisation algorithms is first investigated. It is shown that even for very simple cases, local optimisation based approach may fail to find the worst cases. To overcome this problem, global optimisation algorithms based verification process is investigated in Section 5. Simulation results are presented to verify the proposed verification processes. Finally, Section 6 concludes the paper and outlines some future research directions.

\section{Unicycle Mobile Robot Model}

A schematic figure of a unicycle mobile robot is shown in Fig.1. This type of robot is mostly used for indoor applications. below:

The kinematic equations of the unicycle robot are given

$$
\left[\begin{array}{c}
\dot{x} \\
\dot{y} \\
\dot{\theta}
\end{array}\right]=\left[\begin{array}{cc}
\cos \theta & 0 \\
\sin \theta & 0 \\
0 & 1
\end{array}\right]\left[\begin{array}{l}
v \\
\omega
\end{array}\right]
$$

where $v$ and $\omega$ are linear and angular velocities of the robot. Note that $[v w]^{T}$ defines the inputs of the kinematic system. $(x, y)$ are the robot position coordinates, and $\theta$ represents the orientation of the robot.

The vehicle has two identical parallel, non-deformable rear wheels that are controlled by two independent motors and a steering front wheel.

The dynamic equations of the unicycle mobile robot can be written as

$$
\left[\begin{array}{c}
\dot{v} \\
\dot{\omega}
\end{array}\right]=\left[\begin{array}{cc}
\frac{1}{m} & 0 \\
0 & \frac{1}{J}
\end{array}\right]\left[\begin{array}{l}
F \\
\tau
\end{array}\right]
$$

where $m$ is the robot's mass and $J$ is the inertia moment. $F$ and $\tau$ are the forward force input and moment torque input applied by the wheel motors, respectively ${ }^{[9,17]}$.

\section{Motion Control and Obstacle Avoidance}

The control system is proposed to have an inner-outer-loop structure (Fig.2). The inner-loop control law is responsible to compute the force and torque signals that will drive the wheel's motors to force the robot to move according to a desired linear and angular velocity. These desired velocities are the control signals generated by the outer-loop controller ${ }^{[10]}$.

\subsection{Inner-Loop Controller}

To accomplish the goal of driving the robot to a desired linear velocity $v_{d}$ and angular velocity $\omega_{d}$, a first step is to compute the error between the true velocities and the desired ones. To this effect, let $e_{v}=v_{d}-v$ and $e_{\omega}=\omega_{d}-\omega$ be respectively the linear and angular velocity errors. A simple proportional control law is proposed as speed controllers.

$$
\begin{aligned}
F & =K_{1} e_{v} \\
\tau & =K_{2} e_{\omega}
\end{aligned}
$$

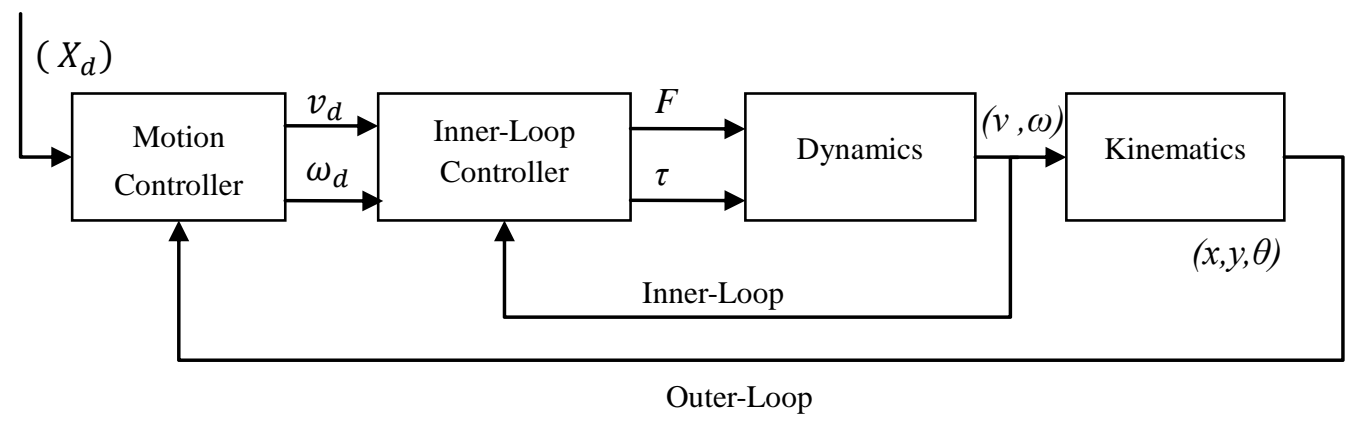

Figure 2. Model of the mobile-robot including kinematics, dynamics and the controllers. 


\subsection{Outer-Loop Controller}

The incremental motion planning for nonholonomic robot is considered in this section. In general, a kinematic model is used for motion planning and collision avoidance.

The kinematic model of the wheeled mobile robot (1) can be represented in a general state space form as

$$
\dot{X}=G(X) u
$$

where $X \in \mathbb{R}^{n}$ is the vector of generalized coordinates, and $u \in \mathbb{R}^{m}(m<n)$ is the control input vector.

Given any desired smooth trajectory $X_{d}(t)$, a straightforward approach is to determine the input command $u$ using the pseudo-inverse control law

$$
u=G^{\#}(X) \dot{X}_{d}
$$

where

$$
G^{\#}(X)=\left[G^{T}(X) G(X)\right]^{-1} G^{T}(X)
$$$$
\text { is }
$$

the

pseudo-inverse of $G(X)$.

This solution locally minimizes the error $\left(\dot{X}_{d}-\dot{X}\right)$ in a least-squares sense ${ }^{[11,12]}$. If the desired velocity $\dot{X}_{d}$ is feasible at the current $X$, the control law in (5) results in zero velocity error. Note that the pseudo-inverse gives the command input $u$ as a feedback law depending on the current state $X^{[11,12]}$

For the unicycle robot, $X=(x, y, \theta)$ is the configuration vector. Comparing to (1) and (4),

$$
G(X)=\left[\begin{array}{cc}
\cos \theta & 0 \\
\sin \theta & 0 \\
0 & 1
\end{array}\right]
$$

Let $u=\left[u_{1}, u_{2}\right]^{T}$, where $u_{1}$ is the linear velocity and $u_{2}$ is the angular velocity.

It follows from (5) that the pseudo-inverse of $G(X)$ takes the form

$$
G^{\#}(X)=\left[\begin{array}{ccc}
\cos \theta & \sin \theta & 0 \\
0 & 0 & 1
\end{array}\right]
$$

and the feedback law (5) for tracking a desired trajectory $X_{d}=\left(x_{d}, y_{d}, \theta_{d}\right)$ becomes

$$
u=G^{\#}(X) \dot{X}_{d}=\left[\begin{array}{ccc}
\cos \theta & \sin \theta & 0 \\
0 & 0 & 1
\end{array}\right]\left[\begin{array}{c}
\dot{x}_{d} \\
\dot{y}_{d} \\
\dot{\theta}_{d}
\end{array}\right]
$$

Therefore, the resulting input command will be

$$
\begin{gathered}
u_{1}=v_{d}=k_{p}\left(\dot{x}_{d} \cos \theta+\dot{y}_{d} \sin \theta\right) \\
u_{2}=\omega_{d}=k_{\theta} \dot{\theta}_{d}
\end{gathered}
$$

where gains $k_{p}$ and $k_{\theta}$ are introduced for additional freedom in weighting the two input commands. In order to apply the control law (9-10), the desired values for $\dot{x}_{d}, \dot{y}_{d}$, and $\dot{\theta}_{d}$ are required. $\dot{X}_{d}$ may be determined by a local holonomic planner using the potential field method as described in the next section.

\subsection{Potential Field Method}

The robot motion in the potential field method can be interpreted as the motion of a particle in a gradient vector field generated by positive and negative electric particles. In this analogy, the robot is a positive charge. Gradients in this context can be interpreted as forces that attract the positively charged robot particle to a negative particle that acts as the goal. The obstacles act as positive charges generating repulsive forces that force the particle robot away from the obstacles. The combination of the attractive force to the goal and repulsive forces away from the obstacles drive the robot in a safe path to the goal ${ }^{[13]}$.

Let $q=(x, y)$ denote the position of robot in the potential field. The usual choice for the attractive potential is the standard parabolic that grows quadratically with the distance to the goal,

$$
U_{\text {att }}(q)=\frac{1}{2} k_{a} d_{\text {goal }}^{2}(q)
$$

where $\quad d_{\text {goal }}=\left\|q-X_{\text {goal }}\right\|$ is the Euclidean distance of the robot $q$ to the goal $X_{\text {goal }} . k_{a}$ is a scaling factor. The gradient

$$
\nabla U_{a t t}(q)=k_{a}\left(q-X_{\text {goal }}\right)
$$

The attractive force considered in the potential field based approach is the negative gradient of the attractive potential

$$
F_{a t t}(q)=-\nabla U_{a t t}(q)=-k_{a}\left(q-X_{g o a l}\right)
$$

Setting the robot velocity vector proportional to the vector field force, the force $F_{a t t}(q)$ drives the robot to the goal with a velocity that decreases when the robot approaches the goal. The attractive potential field at target position $(6,7)$ is shown in Fig.3.

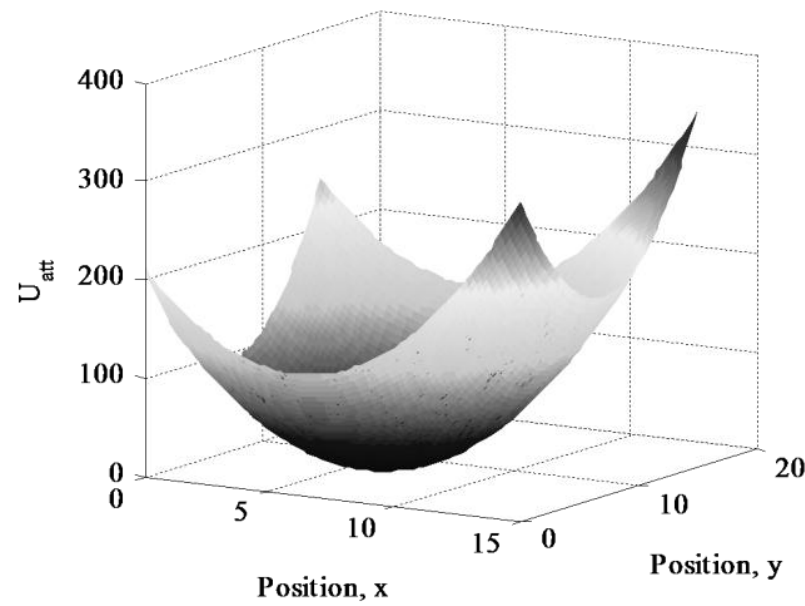

Figure: 3- Attractive Potential field, U_att 
The repulsive potential keeps the robot away from obstacles, which are either a priori known or detected by robot on-board sensors. This repulsive potential is stronger when the robot is closer to the obstacles and has a decreasing influence when the robot is far away. To account for this effect and to the space bounded influence, a possible repulsive potential generated by obstacle $i$ is

$$
U_{\text {rep }_{i}}(q)=\left\{\begin{array}{lll}
\frac{1}{2} k_{r}\left(\frac{1}{d_{\text {obst }}(q)}-\frac{1}{d_{0}}\right)^{2}, & \text { if } & d_{\text {obst }_{i}}(q) \leq d_{0} \\
0 & , \text { if } & d_{\text {obst }_{i}}(q)>d_{0}
\end{array}\right.
$$

where $i$ is the number of obstacle that are close to the robot, $d_{\text {obst }}(q)$ is the closest distance to the obstacle $i$, $k_{r}$ is a scaling constant and $d_{0}$ is the obstacle influence threshold ${ }^{[13]}$. Repulsive potential field $U_{-}$rep is given in Fig.4. The negative gradient of the repulsive potential, $F_{r e p_{i}}(q)=-\nabla U_{r e p_{i}}(q)$, is given by,

$$
F_{\text {rep }_{i}}(q)=\left\{\begin{array}{rr}
k_{r}\left(\frac{1}{d_{\text {obst }_{i}}(q)}-\frac{1}{d_{o}}\right) \frac{1}{d_{\text {obst }}^{2}(q)} \hat{e}_{i}, \text { if }_{\text {obst }_{i}}(q) \leq d_{o} \\
0, & \text { if } d_{\text {obst }_{i}}(q)>d_{o}
\end{array}\right.
$$

where $\hat{e}_{i}=\frac{\partial d_{o b s t}(q)}{\partial(q)}$ is a unit vector that indicates the direction of the repulsive force ${ }^{[5]}$. Therefore,

$$
\left[\begin{array}{c}
\dot{x}_{d} \\
\dot{y}_{d}
\end{array}\right]=-\nabla\left(U_{a t t}(q)+U_{r e p}(q)\right)=F_{a t t}(q)+F_{r e p_{i}}(q)
$$

In order to complete the planning method, the rotational part of $\dot{\theta}_{d}$ is defined. For the unicycle it is convenient to use

$$
\dot{\theta}_{d}=\operatorname{atan} 2\left\{\frac{\dot{y}_{d}}{\dot{x}_{d}}\right\}-\theta
$$

By defining $\operatorname{atan} 2\{0,0\}=0$, the above function remains continuous along any approaching direction to the goal. Therefore, the resulting command $u_{1}$ and $u_{2}$ are determined by the (9-10) using with (16-17).

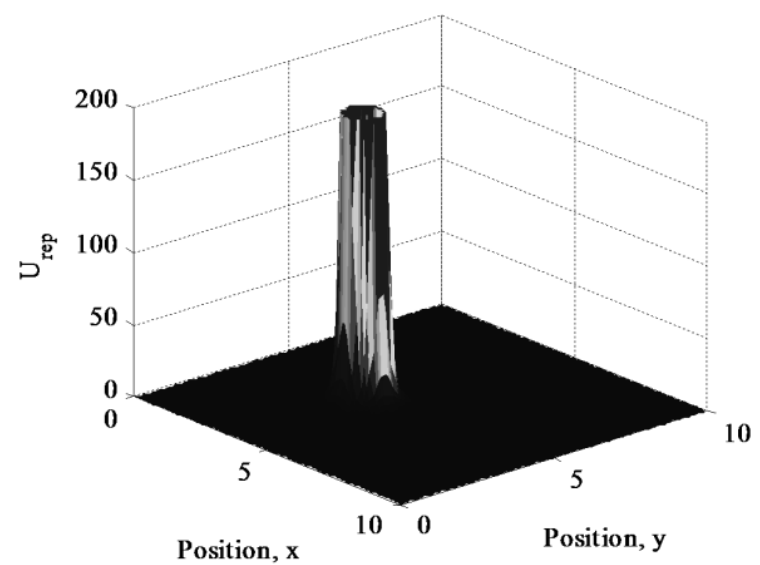

Figure: 4- Repulsive Potential field, U_rep
In this section, the simulation results for a unicycle mobile robot among circular obstacles in a two-dimensional workspace are presented and then the robustness analysis and verification of the proposed algorithm for the unicycle mobile robot will be investigated in the next section. The nominal parameter values are $m=5 \mathrm{Kg}$ and $J=0.05 \mathrm{Kgm}^{2}$. Controller gains are set to $k_{p}=0.06, k_{\theta}=5, K_{1}=6, K_{2}=5$ while the holonomic planner parameters are $k_{a}=5, k_{r}=4$. The influence range $d_{0}$ is chosen as $2 \mathrm{~m}$. The target position is located at $(6,7)$, and the obstacle is located at $(4,4)$ with a safety radius $(r)$ of $0.5 \mathrm{~m}$. The robot starts from initial position $(0$, 0 ). The simulation result is shown in Fig. 6. The minimum distance to the obstacle is obtained as $0.9478 \mathrm{~m}$ which is greater than obstacle safety radius $0.5 m\left(d_{\min }>r\right)$. Therefore, the obstacle avoidance algorithm is working correctly at the nominal parameters. The total potential field $\left(U \_T o t a l=U \_a t t+U_{-} r e p\right)$ is shown in Fig.5.

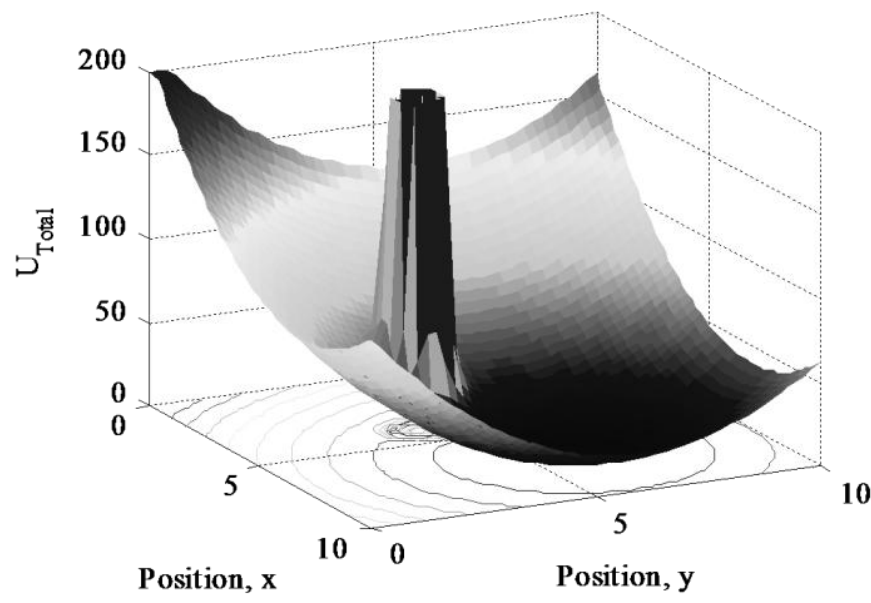

Figure: 5- Total Potential field, U_Total

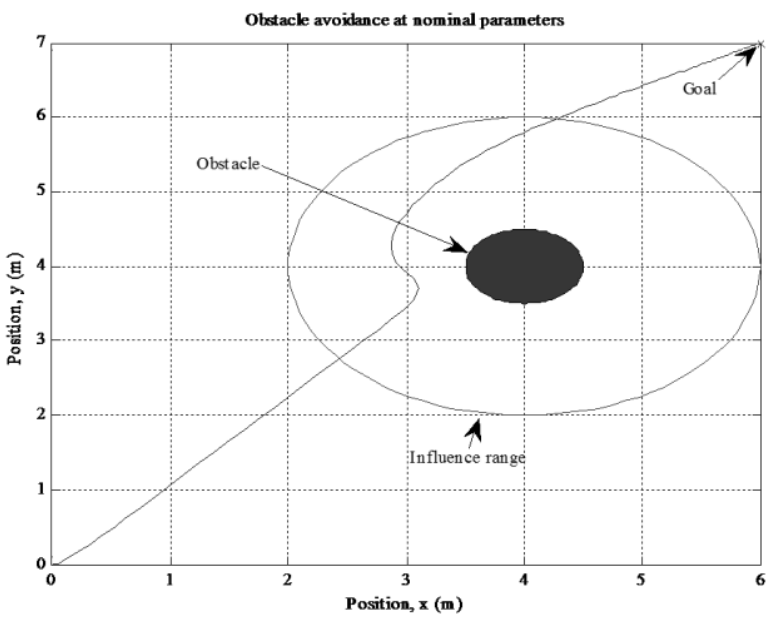

Figure: 6- Simulation result for unicycle robot with obstacle at nominal parameters 


\section{Initial Robustness Analysis and Local Optimisation Method}

\subsection{Initial Robustness Analysis}

Initial robustness analysis of the proposed algorithm is firstly carried. Uncertainties are considered in the dynamic model, and each uncertain parameter is allowed to vary within $\pm 20 \%$ of its nominal value. Two uncertain parameters mass $(m)$ and inertia $(J)$ are firstly considered within lower and upper bounds, i.e. $m=[4,6] \mathrm{Kg}$, and $J=$ $[0.04,0.06] \mathrm{Kgm}^{2}$. Fig. 8 and 9 show variations of the minimum distance to the obstacle with respect to the mass and inertia. There is a small variation in the distance with the variations of the mass, but in a nonlinear form, whereas the minimum distance to the obstacle monotonously decreases with the increase of the inertia.

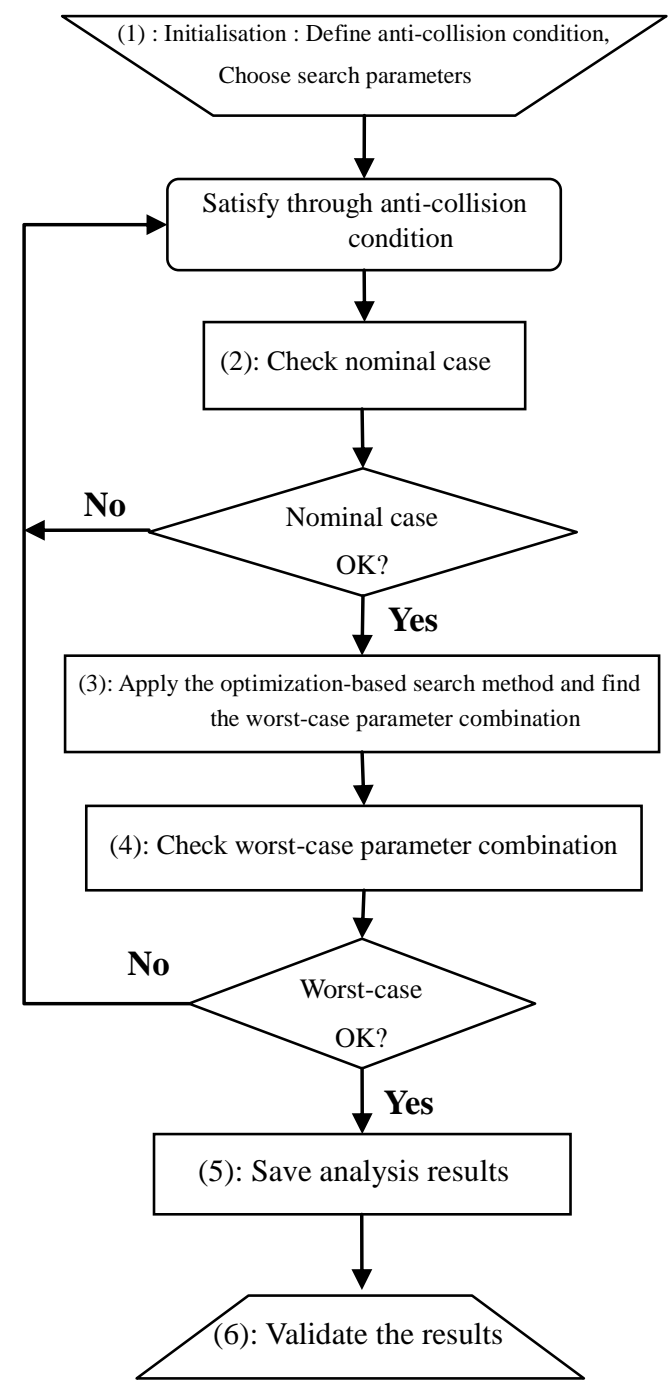

Figure: 7 Optimisation-based verification analysis cycle

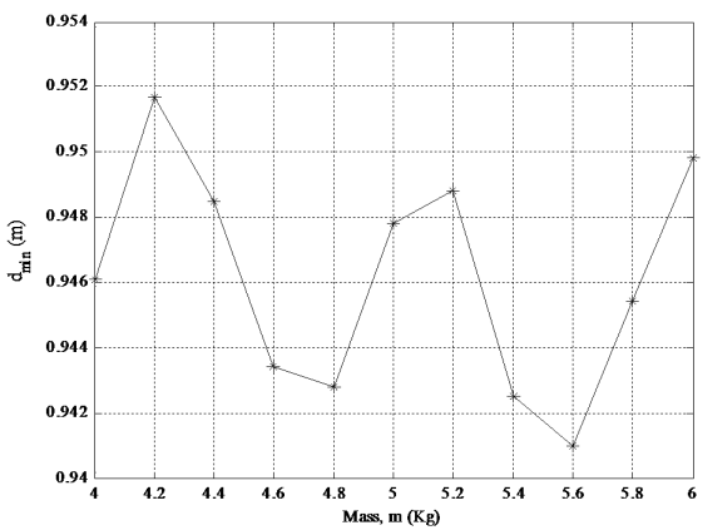

Figure: 8 - Mass variations in $20 \%$ range

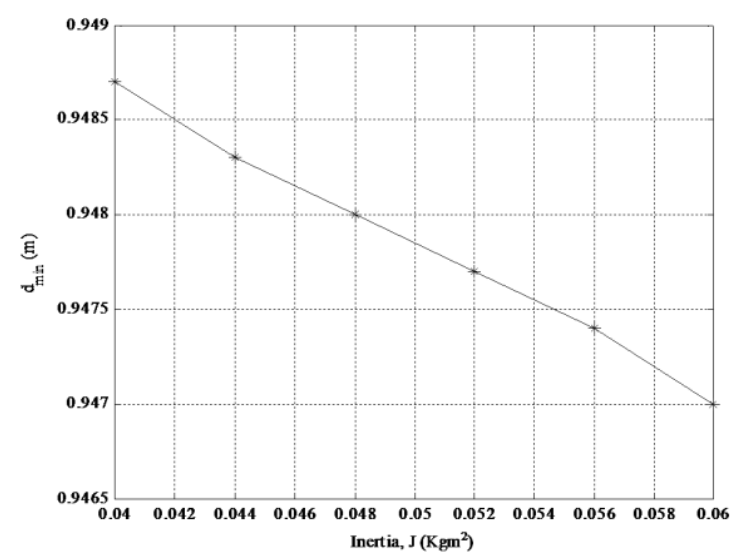

Figure: 9- Inertia J- variations in $20 \%$ range

\subsection{Optimisation-based Worst-Case Analysis}

The optimisation verification process is applied to the unicycle dynamic systems. In the introduction section, the anti-collision avoidance condition is presented for obstacles i.e. if the minimum distance to the obstacle is greater than a specified safe margin $\left(d_{\min }>r\right)$ during the robot moving, then the proposed anti-collision algorithm is safe. When the optimisation verification process is applied to the system, this anti-collision condition is checked for all possible variations. The local and global optimisation methods are applied to the problem of evaluating a worst-case condition and parameters for the unicycle robot collision avoidance systems. Uncertain parameters are considered that lies between given upper and lower bounds. The objective function is

$$
\begin{gathered}
\left.d_{\min }=\min (d(t)) \quad \text { for } t \leq T \text { (sec }\right) \\
\text { s.t } \quad P_{L} \leq P \leq P_{U}
\end{gathered}
$$

where $P$ is the uncertain parameters set. $P_{L}$ and $P_{U}$ are the lower and upper bounds of $P . d(t)$ is the distance to the obstacle $T$ is the collision avoidance manoeuvre during the period and $d_{\text {min }}$ is the minimum distance to the obstacle.

Analysis cycle used for the verification of an obstacle avoidance algorithm is illustrated by the flow diagram in Fig.7. Initialisation is the first step for the obstacle avoidance verification process. Anti-collision 
condition is defined and uncertain parameters are chosen to determine the worst-case. Before applying the optimisation algorithm, the anti-collision condition can be checked at nominal case. If it satisfies, then the optimization methods are applied and determine the worst-case condition and worst-case parameters. Otherwise, the obstacle avoidance algorithm and controller have to redefine to satisfy the anti-collision condition. In order to find the worst case condition and worst case parameters, several optimisation algorithms are applied to this problem.

\subsection{Local Optimisation-based Worst-Case Analysis}

Sequential Quadratic Programming (SQP) methods are standard general purpose algorithms for solving smooth and well-scaled nonlinear optimisation problems when the functions and gradients can be evaluated with high precision. It is an iterative method starting from an initial point and converging to a local minimum. The function fmincon is a MATLAB implementation. The optimization processing of fmincon consists of three main stages:

(i) updating of the Hessian matrix of the Lagrangian function, (ii) quadratic programming problem solution, and (iii) line search and merit function calculation. This iteration is repeated until an optimal or feasible solution is found ${ }^{[19]}$. The local optimisation method is applied with different starting points to the problem of evaluating a clearance criterion for the unicycle robot obstacle avoidance systems.

This iteration is repeated until a specified termination criterion (either maximum number of function evaluations or convergence accuracy) is met. The results of the minimum distance to the obstacle and worst case parameters with different starting points are given in Table.1. The results clearly show that fmincon does not give the same solutions for this problem, because a local optimization algorithm depends on the starting point. Fig.10 shows the worst-case violation of the optimal solution. Therefore, global optimisation methods are applied to find the true worst-case.

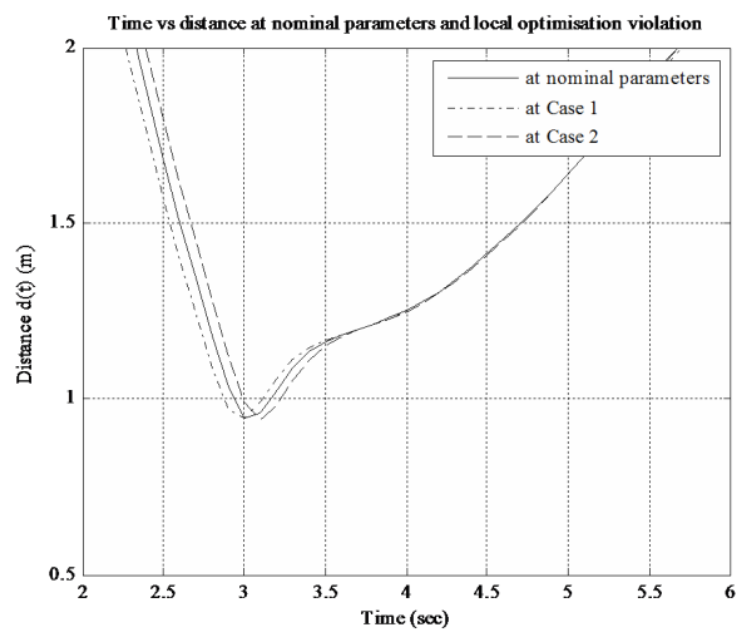

Figure.10. fmincon Worst-case violation
TABLE.1 LOCAL OPTIMISATION RESULTS

\begin{tabular}{cccc}
\hline fmincon & Starting point & Convergent point & $d_{\text {min }}(m)$ \\
\hline Case-1 & {$[4.5,0.05]$} & {$[4.7179,0.06]$} & $\mathbf{0 . 9 4 1 3}$ \\
Case-2 & {$[5.5,0.048]$} & {$[5.5487,0.06]$} & 0.9397 \\
\hline
\end{tabular}

\section{Global Optimisation-based Worst-Case Analysis}

\subsection{Genetic Algorithms}

Genetic Algorithms (GA's) are general purpose stochastic search and optimisation algorithms, based on genetic and evolutionary principles. The theory and practice of the GA was originally invented by John Holland in the 1960s and was fully elaborated in his book Adaption in Natural and Artificial Systems which was published in $1975^{[18]}$. The basic idea of the approach is to start with a set of designs, randomly generated using the allowable values for each design variable. Each design is also assigned a fitness value. The process is continued until a stopping criterion is satisfied or the number of iterations exceeds as a specified limit. Three genetic operators are used to accomplish this task: Selection, Crossover, and Mutation. Selection is an operator where an old design is copied into the new population according to the design's fitness. There are many different strategies to implement this selection operator including roulette wheel selection, tournament selection and stochastic universal sampling. The crossover operator corresponds to allowing selected members of the new population to exchange characteristics of their designs among themselves. Crossover entails selection of starting and ending positions on a pair of randomly selected strings, and simply exchanging the string of 0's and 1's between these positions. Mutation is the third step that safeguards the process from a complete premature loss of valuable genetic material during selection and crossover. The foregoing three steps are repeated for successive generations of the population until no further improvement in fitness is attainable $\left.{ }^{[8,14,15]}\right]$.

GA can be applied to the unicycle collision avoidance system to find the global minimum. The uncertain parameter set is considered here as the genetic representation, i.e. the chromosome. Each of the uncertainties corresponds to one gene. A binary coded string is generated to represent the chromosome, where each of the uncertain parameters lies between the lower and upper bounds. The selection function of stochastic uniform is used for this example. The population size and crossover fraction are selected as default value of 20 and 0.8 respectively. The level of accuracy for each parameters is chosen as $10^{-6}$. The process is continued until a number of iterations exceeds as a specified limit (50). The GA 
results with different starting points are given in Table.2. Fig. 11 shows the number of generations versus the best fitness and the mean fitness values at starting point [4.5, 0.05]. GA gives the unique worst-case $d_{\min }$ and worst-case parameter set solution with different starting points for this problem.

\subsection{GLOBAL Algorithm}

The multistart clustering algorithm presented in this work is based on GLOBAL (Csendes, 1988), which is a modified version of the stochastic algorithm by Boender et al (1982) implemented in FORTRAN ${ }^{[16]}$. The GLOBAL method has two phases i.e. a global and a local one. The global phase consists of sampling and clustering, while the local phase is based on local searches. A general clustering method starts with the generation of a uniform sample in the search space (the region defined by lower and upper bounds). After transforming the sample (by selecting a user set percentage of the sample points with the lowest function values), the clustering procedure is applied. Then, the local search is started from those points which have not been assigned to a cluster. GLOBAL uses the Single Linkage clustering rule ${ }^{[16]}$.

The new implementation GLOBALm, which has been written in MATLAB, is freely available for academic purposes ${ }^{[16]}$. It is the bound constrained global optimisation problems with black-box type objective function. GLOBALm has different local optimisation methods which are capable of handling constraints. The UNIRANDI local search method is part of GLOBAL package while the BFGS (Broyden-Fletcher-Goldfarb-Shanno) local search is part of the MATLAB package. GLOBAL has its own termination criteria, so it stops when did not find any new local minimum and all the sample points were clustered. It also stops when the number of find local minimum exceeds a given values. GLOBAL is a direct search method because the user must not include subroutines for the calculation of derivatives only that for the objective function itself.

The GLOBAL optimisation with UNIRANDI local search method is applied to find the global solution for unicycle obstacle avoidance system. Maximum number of function evaluations for local search is chosen as a default value of 1000 . The results with different sampling points are given in Table.3. In the table, the last column shows the number of function evaluation taken for this simulation with different sampling points.
It is interesting to note that when the sampling points are increased, then function evaluations taken are reduced. GLOBAL algorithm is also converged to a unique solution. Therefore, these two global optimization methods perform well for this problem.

Final step of the analysis process is to validate the proposed algorithm results. Therefore, these worst-case condition and worst-case parameters are further validated using with simulation response which is shown in Fig.12. The worst-case minimum distance to the obstacle $d_{\min }$ is $0.9397 \mathrm{~m}$ which is greater than the safety margin. This response shows that the obstacle avoidance algorithm and the controller are working correctly at worst-case parameters. And also, the time versus distance to the obstacle at nominal and worst-case parameters is shown in Fig.13. At worst-case parameters, the simulation response with two obstacles is shown in Fig.14. Therefore, the proposed controller for one obstacle is functioning correctly for two obstacles at worst-case parameters.

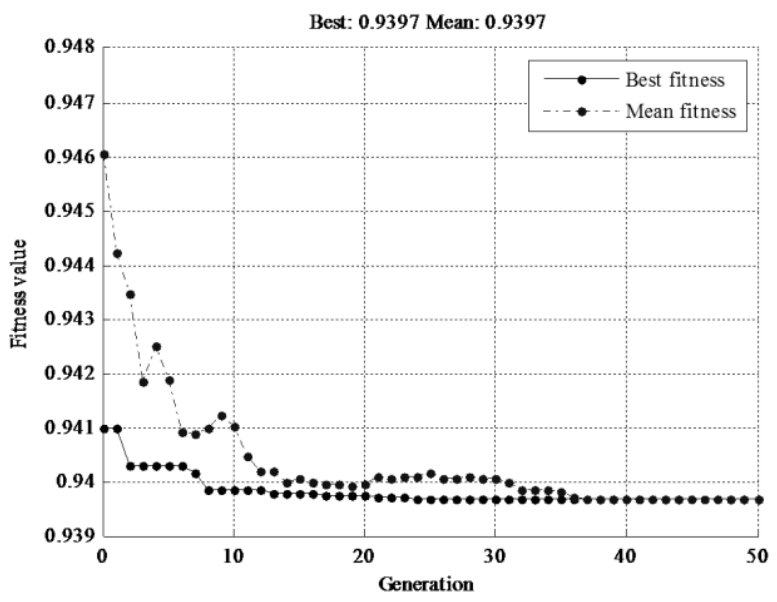

Figure.11. No of generations vs. Fitness value

TABLE.2 GA RESULTS FOR A UNICYCLE OBSTACLE AVOIDANCE SYSTEM

\begin{tabular}{ccccc}
\hline Algorithm & Starting point & $m(K g)$ & $J\left(K^{2} m^{2}\right)$ & $d_{\min }(m)$ \\
\hline$G A$ & {$\left[\begin{array}{llll}5, & 0.05\end{array}\right]$} & 5.5487 & 0.06 & 0.9397 \\
$G A$ & {$[4.5,0.05]$} & 5.5487 & 0.06 & 0.9397 \\
\hline
\end{tabular}

TABLE.3 GLOBAL RESULTS FOR UNICYCLE OBSTACLE AVOIDANCE SYSTEM

\begin{tabular}{lccccc}
\hline Algorithm & No of SAMPLE & $m(\mathrm{Kg})$ & $J\left(\mathrm{Kgm}^{2}\right)$ & $d_{\min }(\mathrm{m})$ & Fun.Evalu taken \\
\hline GLOBAL-with UNIRANDI & 20 & 5.5489 & 0.06 & 0.9397 & 366 \\
GLOBAL-with UNIRANDI & 50 & 5.5487 & 0.06 & 0.9397 & 298 \\
\hline
\end{tabular}




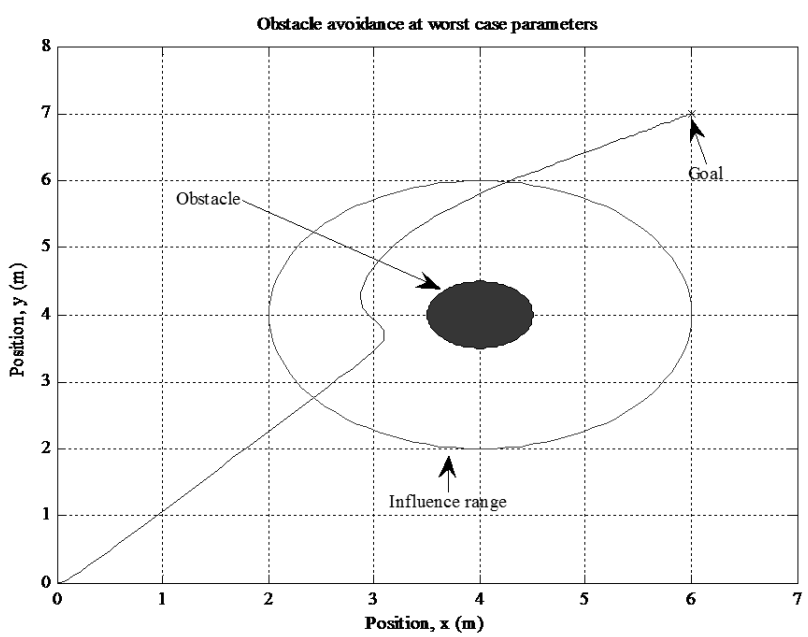

Figure.12. Simulation response at worst- case parameters

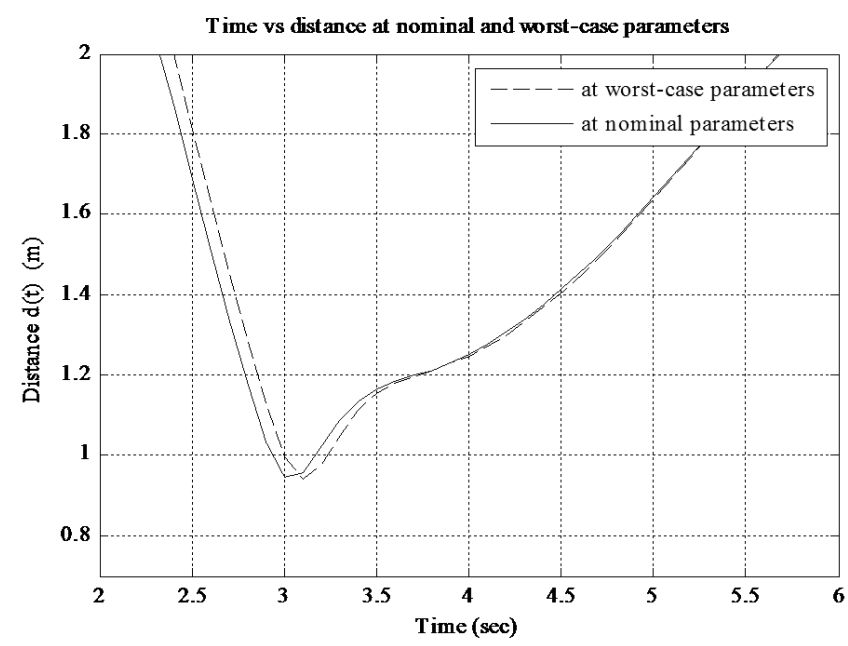

Figure.13: Time vs distance to the obstacle at nominal and worst-case parameters

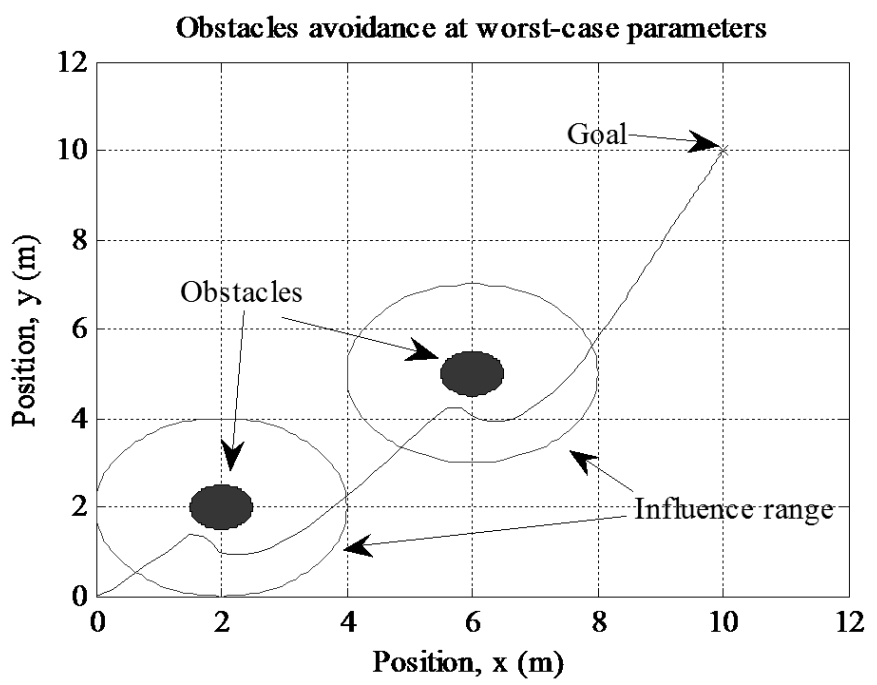

\section{Conclusions}

In this paper, the safety analysis of collision avoidance systems is presented. The optimization-based verification process method is applied for verification of collision avoidance algorithms for an unicycle robot. First, kinematic and dynamic equations of the unicycle robot are introduced and the controller is presented based on these equations. The inner-outer-loop control architecture is used where the inner-loop controller is a proportional speed controller. A local planner in the outer-loop is developed using the artificial potential field method. Then an optimisation based approach is developed to find the worst cases which are defined by the minimum distance to the obstacle in the presence of all possible described variations. Mass and inertia variations are considered in this case study. The local optimisation method does not give the unique solution. It clearly shows that the optimal solutions do not converge to the global minimum. Different worst cases are identified when the optimisation starts from different initial conditions. Therefore, the local optimisation is not suitable for verification of collision avoidance algorithms for this case study. As demonstrated by Fig.8 and Fig.9, it is a non convex nonlinear optimisation problem and it is possible to miss the worst case. To overcome this problem, global optimisation algorithms are required.

Global optimisation including GA and GLOBAL methods are applied to the problem of analysing the robustness properties of a unicycle dynamic system. Both these two algorithms perform well for this example. However, these are stochastic global optimization algorithms. Further work will be developing deterministic global optimization-based worst-case analysis and applying the proposed approach to more complicated unmanned vehicles in particular Unmanned Aerial Vehicles (UAVs). 


\section{References}

[1]. Farbod Fahimi, C.Nataraj and Hashem Ashrafiuon, "Real-time obstacle avoidance for multiple mobile robots”, Robotica (2009), Vol-27, pp 189-198, 2008.

[2]. O.Khatib, "Real-time obstacle avoidance for manipulators and mobile robots", Int. Journal Robotics Research, Vol.5, No-1, pp,90-99, 1986.

[3]. Jasmin Velagic, Bakir Lacevic and Nedim Osmic, "Efficient path planning algorithm for mobile robot navigation with a local minima problem solving", IEEE International Conference on Industrial Technology, 2006.

[4]. S.S.Ge and Y.J.Cui, “ Dynamic motion planning for mobile robot using potential filed method", Autonomous Robots (13), 207-222, 2002.

[5]. Eliana Costa e Silva, E. Bicho, W. Erlhagen, "The potential field method and the nonlinear attractor dynamics approach: what are the difference?", $7^{\text {th }}$ Portuguese Conference on Automatic Control", CONTROLO 2006.

[6]. C.Fielding, A.Varga, S.Bennai and M.selier(Eds), "Advanced techniques for clearance of flight control laws", Springer LNCIS 283, Heidelberg 2002.

[7]. K.K. James, Lexington, "Safety analysis methodology for unmanned aerial vehicle (UAV) collision avoidance systems", United States Air Force, \#F19628-00-C-0002.

[8]. W.M. Rand, "Controlled observations of the Genetic Algorithm in a changing environment: case studies using the Shaky ladder hyperplane-defined functions", Thesis, The University of Michigan, 2005.

[9]. J. Wang, X. Wu, Z. Xu, "Potential-based obstacle avoidance in Formation Control", J Control Theory April 20086 (3) 311-316.

[10]. R. Carona, A. P. Aguiar, J. Gaspar, "Control of unicycle type robots", In Proc. Of iv Jornadas de Engenharia Electronica e Telecomunicacoes, pp 180-185, Portugal, Nov 2008.

[11]. A.D. Luca, G. Oriolo, "Local Incremental Planning for Nonholonomic Mobile Robots", Proceeding.of.IEEE International Conference on Robotics and Automation, 1994.

[12]. A. Bemporad, A.D. Luca, G. Oriolo, "Local Incremental Planning for a car-like robot navigating among obstacles", Proceedings of IEEE International Conference on Robotics and Automation, 1996.

[13]. Mario Isabel Ribeiro, "Obstacle avoidance", Instituto de Sistemas e Robótica, November 2005, http://users.isr.ist.utl.pt/ mir/
[14]. D. Bates, M. Hagstrom, "Nonlinear analysis and synthesis techniques for aircraft control", Springer, 2007.

[15]. J. S. Arora, "Introduction to optimum design", second Edition, Elsevier Academic Press.

[16]. T.Csendes, L.Pal, J.O.H.Sendin, J.R.Banga "The GLOBAL optimisation method Revisited", University of Szeged, Hungary, Sept, 2008.

[17]. V.Gazi, B.Fidan, Y.S.Hanay, M.I.Koksal, "Aggregation, foraging, and formation control of swarms with nonholonomic agent using potential functions and sliding mode techniques", Turkey Journal of Electrical Engineering, Vol.15, No.2. 2007.

[18]. J.Holland, "Adaptation in natural and artificial Systems", University of Michigan press, Ann Arbor, MI, 1975 .

[19]. Mathworks.com

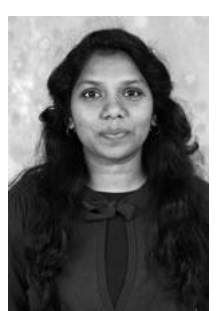

Sivaranjini Srikanthakumar received a B.Eng in Computer Systems Engineering (2007) from Electronics and Electrical Engineering Department, Loughborough University, UK. She currently is a PhD student in the Department of Aeronautical and Automotive Engineering at Loughborough University, UK.

Her research interests include the autonomous vehicles verification, Optimisation-based clearance process, Collision avoidance algorithm, path planning, global optimisation methods and flight control laws.

E-mail: sivaranjinisk@yahoo.co.uk

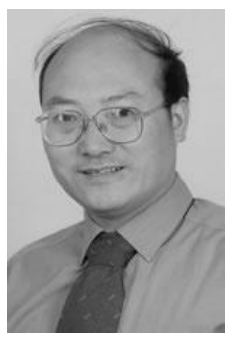

Wen-Hua Chen received his M.Sc and Ph.D. degrees from Department of Automatic Control at Northeast University, China, in 1989 and 1991, respectively. From 1991 to 1996, he was a lecturer in Department of Automatic Control at Nanjini Universuty of Aeronautics and Astronautics, China. He held a research position and then a lectureship in control engineering in Center for Systems and Control at University of Glasgow, UK, from 1997 to 2000. He currently holds a senior lectureship in flight control systems in Department of Aeronautical and Automotive Engineering at Loughborough University, UK.

He has published one book and more than 100 papers on journals and conferences. His research interests include the development of advanced control strategies and their applications in aerospace engineering.

E-mail:W.Chen@lboro.ac.uk 